\title{
Assessment of longitudinal strain in the Carotid artery wall using ultrasound-based Speckle tracking - validation in a sheep model
}

\author{
Matilda Larsson ${ }^{1,3}$, Peter Verbrugghe ${ }^{2}$, Marija Smoljkić ${ }^{3}$, Brecht Heyde ${ }^{2}$, Nele Famaey ${ }^{3}$, \\ Paul Herijgers ${ }^{2}$, Jan D'hooge ${ }^{2}$ \\ ${ }^{1}$ Department of Medical Engineering, Royal Institute of Technology - KTH, Stockholm, Sweden \\ ${ }^{2}$ Department of Cardiovascular Sciences, KU Leuven, Leuven, Belgium \\ ${ }^{3}$ Biomechanics Section, Faculty of Engineering, KU Leuven, Leuven, Belgium \\ matilda.larsson@sth.kth.se
}

\begin{abstract}
Assessment of strain in the longitudinal direction of the arterial wall has been suggested to improve the evaluation of arterial stiffness and atherosclerosis. Recently, we showed the feasibility of ultrasound speckle tracking to assess carotid longitudinal strain in-silico and in-vitro. However, validation in the more challenging in-vivo setting is still lacking. The aim of this study was to validate longitudinal strain assessment in the common carotid artery (CCA) in an animal setup. The left CCAs of five sheep were exposed during Isoflurane anesthesia and sonomicrometry crystals were sutured onto the artery wall to obtain reference longitudinal strain. Ultrasound long-axis images were recorded at baseline and hypertension (Phenylephrine) and an in-house speckle tracking algorithm was applied to estimate longitudinal strain. The estimated strain curves varied cyclically throughout the cardiac cycles, showing a lengthening of the arterial segment in systole. A significant correlation between peak systolic estimated and reference strain was found $(\mathrm{r}=0.95, \mathrm{p}<0.001)$. The results indicate the feasibility of arterial longitudinal strain assessment in-vivo using ultrasound speckle tracking.
\end{abstract}

Keywords - Ultrasound Speckle tracking; Sheep model; Carotid artery; Longitudinal strain; Sonomicrometry

\section{INTRODUCTION}

Methods for early detection and risk stratification in cardiovascular diseases are of major importance for prevention of acute ischemic events such as stroke and myocardial infarction. In recent years, ultrasound-based methods for estimation of arterial wall movement and strain have gained interest in the assessment of arterial stiffness and atherosclerosis. A variety of methods enabling measurements of radial and circumferential distention and strain have been developed and applied both in phantom setups and in-vivo [1-3] and arterial strain has shown potential in both subclinical detection of increased arterial stiffness [1] and in assessment of plaque characteristics to predict plaque rupture [4].

Assessment of longitudinal movement (along the flow direction) of the arterial wall has been suggested to improve the evaluation of arterial stiffness and atherosclerosis. However, in contrast to radial motion the longitudinal motion of the arterial wall has for long been neglected and remains difficult to assess due to its low amplitude in combination with low spatial resolution. Improved imaging techniques have allowed to estimate arterial longitudinal movement using ultrasound speckle tracking approaches [5, 6] and it has been demonstrated to be of the same magnitude as the radial movement [7]. Moreover, it has been shown that the longitudinal motion of the artery may be an important factor in early detection of cardiovascular diseases [8]. In addition, recent studies associate reduced longitudinal wall motion with plaque burden [9] and potential to predict cardiovascular outcome [10]. This indicates the importance of arterial longitudinal function when assessing and evaluating cardiovascular diseases.

However, the number of studies describing methods for longitudinal arterial strain assessment is limited. Recently, we showed the feasibility of ultrasound-based speckle tracking to assess carotid longitudinal strain in-silico [11] and in-vitro [12], but validation in the more challenging invivo setting is still lacking. Accordingly, the aim of this study was to validate longitudinal strain assessment in the common carotid artery in an animal setup.

\section{METHODS}

Longitudinal strain of the common carotid artery of five sheep was estimated by ultrasound speckle tracking and compared with reference strain via sonomicrometry.

\section{A. Animal Preparation}

The experiments obtained ethical approval $\left(\mathrm{EC} \mathrm{n}^{\circ}\right.$ : P160-2010) from the local ethics committee (Animal Ethics Committee of KU Leuven, Leuven, Belgium) and the experiments were performed in concordance to the Guide for the Care and Use of Laboratory Animals published by the U.S. National Institutes of Health (NIH Publication No. 85-23, revised 1996). Five one-year-old female Swifter sheep (44-54 kg) were sedated with an intramuscular injection of Ketamine $(15 \mathrm{mg} / \mathrm{kg})$ and Xylazine $(2 \%$, $0.01 \mathrm{ml} / \mathrm{kg}$ ). Anesthesia was induced with Isoflurane $(5 \%)$ and the trachea of the animal was intubated. Anesthesia was maintained throughout the procedure with an Isoflurane $(2-4 \%)$ - oxygen $(50 \%)$ mixture, mechanically ventilated at a respiratory rate of 12 breaths/minute. An intravenous line was inserted in an extremity vein and an arterial line in the 
ear to allow drug administration and pressure measurements, respectively. Heart rate, blood pressure, endtidal $\mathrm{CO}_{2}$, and blood $\mathrm{O}_{2}$-saturation were constantly monitored throughout the procedure.

The animal was positioned in supine position on a surgical table with a neck extension of $180^{\circ}$. A $5 \mathrm{~cm}$ wide longitudinal incision was made to expose the left common carotid artery approximately $2.5 \mathrm{~cm}$ distal to the carotid bifurcation, see Fig. 1b. A digital sonomicrometry system (Sonometrics Corporation, London, ON, Canada) was used to obtain reference values of longitudinal strain. Five sonomicrometry crystals with a diameter of $1 \mathrm{~mm}$ were attached with suture Prolene 5/0 to the adventitia of the proximal and distal carotid artery wall according to Fig. 1ab. The incision was then closed in layers and filled with ultrasound gel and saline solution. Later, to obtain a range of longitudinal strain values for the validation, hypertension was induced with infusion of Phenylephrine ( $3 \mu \mathrm{g} / \mathrm{kg} . \mathrm{min})$.

\section{B. Data Acquisition}

Ultrasound and sonomicrometry data were collected at baseline and induced hypertension after wound closure. Ultrasound long-axis images of the left common carotid artery were acquired using a Vivid7 system (GE Healthcare, Horten, Norway) with a linear array transducer (12L). Images were acquired throughout three cardiac cycles in a plane just parallel to the sonomicrometry crystals at a frame rate of 45.3 frames/s with a line density of 13.9 lines $/ \mathrm{mm}$. The image depth was $30 \mathrm{~mm}$, whereas the image width was $27 \mathrm{~mm}$. Fig. 1c shows an example of a grayscale long-axis image of the left common carotid artery in one of the animals. Electrocardiogram (ECG) was registered using needle electrodes connected to the ultrasound system. Data for reference strain calculation were acquired using the sonomicrometry system at a sampling rate of $1 \mathrm{kHz}$ throughout six cardiac cycles. Only one of the systems was active simultaneously in order to avoid sound interference.

\section{Data Analysis}

The collected data were analyzed offline using in-house developed Matlab software. Speckle tracking analysis was performed on the ultrasound images and the inter-crystal distances were processed to assess values of reference longitudinal strain.

\section{Speckle Tracking Analysis}

Speckle tracking analysis was performed on the envelopedetected data throughout three consecutive cardiac cycles using the previously developed algorithm [13]. Twodimensional (2D) motion estimation was performed across subsequent frames using normalized cross-correlation (kernel width: 5 wavelengths $(\lambda)$, kernel length: $2 \lambda, 40 \%$ axial and lateral overlap, spline interpolation for detection of sub-sample motion). To remove outliers, the motion estimates were filtered by applying a $2 \mathrm{D}$ median filter on a region of $0.08 \mathrm{~mm}$ (axial) by $0.3 \mathrm{~mm}$ (lateral). This was followed by linear interpolation between samples to obtain motion estimates in the entire image. The displacement

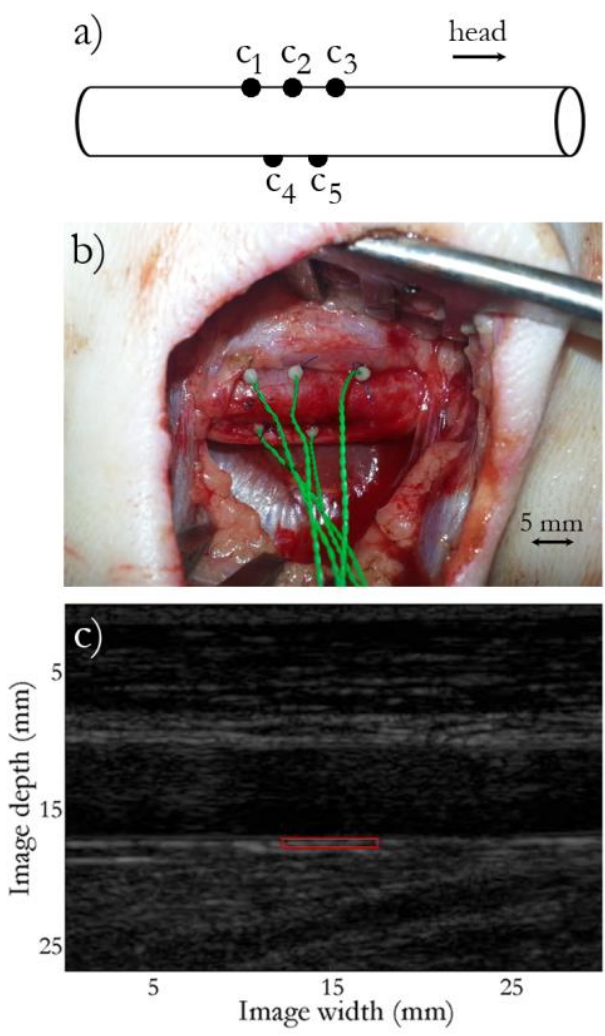

Figure 1. a) Schematic illustration of the left CCA with positions of sonomicrometry crystals (solid circles $\mathrm{c}_{1}-\mathrm{c}_{5}$ ). b) Photo of exposed left CCA with five attached sonomicrometry crystals. c) Ultrasound long-axis image of left CCA with red box indicating ROI for strain analysis. CCA; common carotid artery, ROI; region of interest.

maps were then cumulated throughout the cardiac cycle using linear interpolation to account for sub-pixel motion.

Longitudinal strain was estimated in a region of interest (ROI) manually positioned in the posterior vessel wall close to the position of the crystals (ROI width: $5.4 \mathrm{~mm}$ and ROI length: $0.5 \mathrm{~mm}$ ), see Fig. 1c. The strain values were obtained by spatial linear regression after averaging in the radial direction. Finally, the estimated strain curves were drift compensated to obtain values of zero strain at the end of each cardiac cycle and low-pass filtered in order to remove noise, by convolving the curves with a normalized rectangular function of 6 samples in length.

\section{Sonomicrometry}

The inter-crystal displacement between crystal $c_{4}$ and $c_{5}(D)$ was processed to obtain reference longitudinal strain (Fig. 1a). Other crystals shown in Fig. 1a were not used in this study but enable assessment of e.g. reference circumferential strain in future studies. D was median filtered with a filter length of $35 \mathrm{~ms}$ to reduce noise and then averaged over six cycles. Longitudinal strain $(\varepsilon)$ was then calculated throughout one cycle as:

$$
\varepsilon_{t}=\left(\frac{D_{t}-D_{0}}{D_{0}}\right)
$$




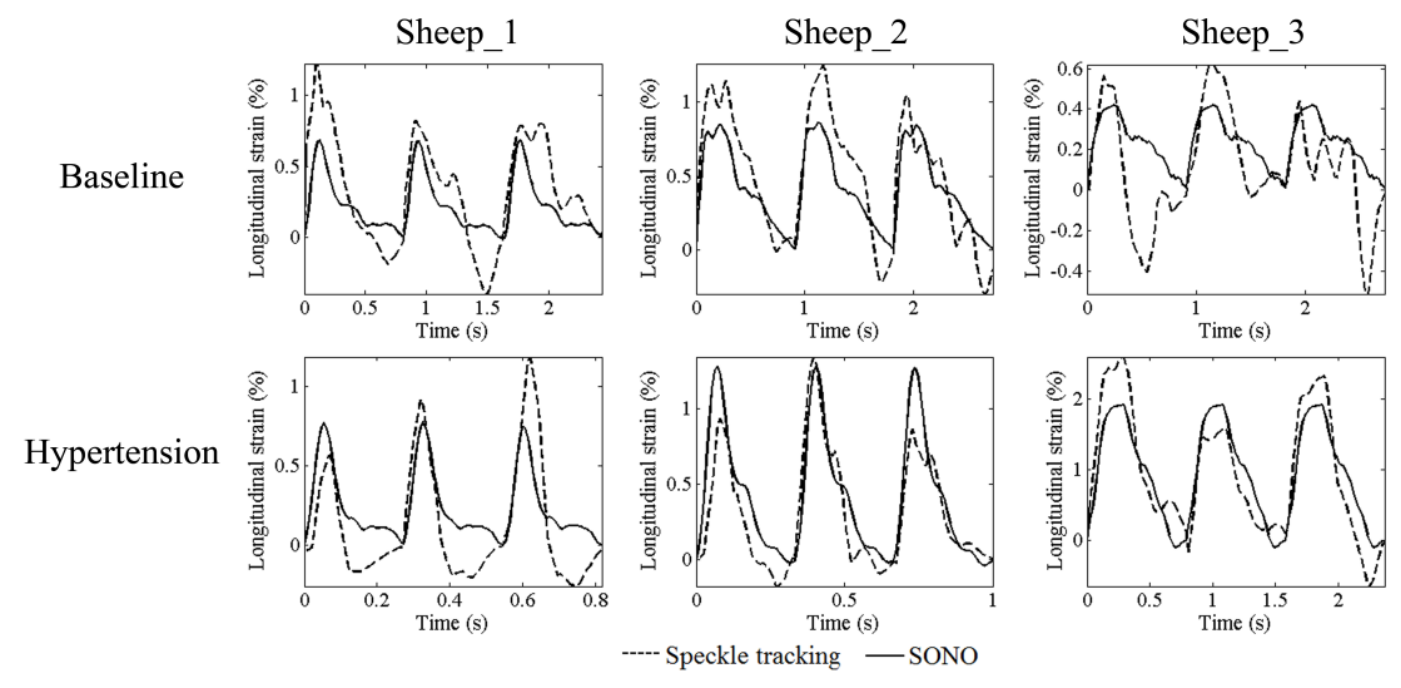

Figure 2. Longitudinal strain curves from the left common carotid artery in a sheep model estimated by speckle tracking and SONO at baseline (upper plots) and hypertension (lower plots). SONO; sonomicrometry.

where $\mathrm{D}_{0}$ was the inter-crystal displacement at the start of the cardiac cycle and $D_{t}$ the inter-crystal displacement at time point $t$. The start of the cardiac cycle was manually identified as the time point before the largest positive derivative of the curve.

\section{Speckle tracking versus Sonomicrometry}

The estimated peak systolic longitudinal strain at baseline and hypertension averaged over three cardiac cycles by speckle tracking was correlated with the reference strain by sonomicrometry using the Pearson correlation coefficient. P-values lower or equal to 0.05 were considered significant. The data was also processed in a Bland-Altman analysis. The variability in strain values over time were calculated as the relative mean difference (RMD) between the three peak systolic strain values as:

$$
R M D=\frac{\left(\left|\varepsilon_{1}-\varepsilon_{2}\right|+\left|\varepsilon_{1}-\varepsilon_{3}\right|+\left|\varepsilon_{2}-\varepsilon_{3}\right|\right)}{3 \times \varepsilon_{\text {sono }}} \times 100
$$

where $\varepsilon_{1-3}$ was peak systolic strain for each of the three cardiac cycles and $\varepsilon_{\text {SONO }}$ the peak systolic reference strain.

\section{RESULTS}

The heart rate was $70 \pm 4 \mathrm{bpm}$ and $142 \pm 57 \mathrm{bpm}$ and the systolic/diastolic blood pressure was $82 \pm 8 / 63 \pm 11 \mathrm{mmHg}$ and $166 \pm 20 / 138 \pm 27 \mathrm{mmHg}$ at baseline and hypertension, respectively. The estimated longitudinal strain curves varied cyclically throughout the cardiac cycle, showing a lengthening of the arterial segment in systole. Longitudinal strain curves from three of the sheep are shown in Fig. 2. A significant correlation between peak systolic estimated and reference strain was found $(\mathrm{r}=0.95, \mathrm{p}<0.001)$ and the mean error between peak systolic strain by speckle tracking and sonomicrometry was $0.14 \pm 0.14 \%$, see Fig. 3. The variability of peak systolic strain over the three cardiac cycles expressed as RMD was $27 \pm 12 \%$.

\section{DISCUSSION}

The results of this study indicate the feasibility of longitudinal strain assessment in the carotid artery wall invivo using ultrasound speckle tracking, since a strong correlation $(\mathrm{r}=0.95, \mathrm{p}<0.001)$ and small differences $(0.14 \pm 0.14 \%)$ were found when comparing with reference strain via sonomicrometry. These results confirm previous studies reporting the feasibility of estimating longitudinal strain of the carotid artery wall both in-silico [11] and invitro [12].

In this study, the investigated arterial segment showed a lengthening in systole, which is in concordance with previous results from video microscopy in rats [14] but contradictive to a previous study using sonomicrometry in a porcine model [15]. In the latter, a shortening of the carotid artery in systole was demonstrated. However, differences in experimental conditions and procedures to attach the crystals are probable reasons to the differing findings.

The surgical exposure may have affected the elastic properties of the artery although the adventitia was left in place and attention was paid to not damage it when suturing the crystals. Still, the obtained longitudinal strain pattern may be different from that of an unexposed artery. Hypertension was induced to alter the arterial strain pattern in order to obtain a range of strain values for validation. However, hypertension did not increase arterial longitudinal strain consistently (Fig. 2). The administration of an alternative drug to induce hypertension may have induced larger longitudinal strains than Phenylephrine, since arterial longitudinal motion has showed to increase in response to catecholamines [16]. However, the inner-most layer of the artery wall showed to increase more in longitudinal motion than the adventitia, resulting in an increased longitudinal shear strain. This indicates that it would most likely not have induced larger strains in this study where the crystals were sutured on the adventitia. 

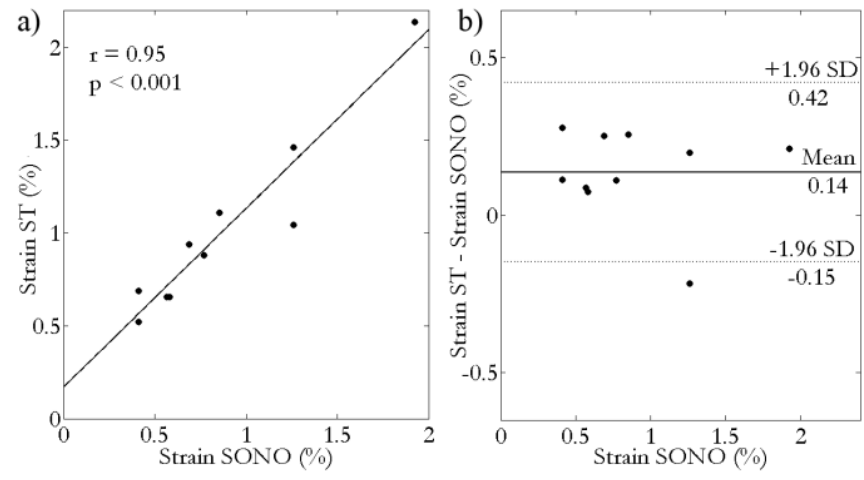

Figure 3. Correlation plot (a) and Bland-Altman plot (b) of peak systolic longitudinal strain by SONO and speckle tracking (mean of three cardiac cycles) in five sheep at baseline and hypertension. ST; speckle tracking, SONO; sonomicrometry

A common source of error in ultrasound speckle tracking estimations is speckle decorrelation due to out-of-plane motion and tissue compression. It is also worth emphasizing the limitation of using sonomicrometry as reference method, since its accuracy is limited in terms of spatial resolution when measuring such small distances. Another factor that could have influenced the discrepancy between the methods was slightly different measurement sites, which was necessary in order to avoid interference of the crystals in the tracking process.

The number of studies describing longitudinal strain of the arterial wall is limited and further research is required to determine the clinical value of arterial longitudinal motion and strain estimations although some studies have already shown that longitudinal motion may be an important contributor in the evaluation of cardiovascular diseases [9, 10]. Likewise, further studies are needed to test the feasibility of this algorithm to perform cardiovascular risk stratification in large patient populations. The relative high variability in strain values over consecutive cardiac cycles obtained in this study stresses the importance of repeated measurements in future studies.

\section{CONCLUSION}

The results from this validation study indicate the feasibility of longitudinal strain assessment in the arterial wall in-vivo using ultrasound speckle tracking. However, the feasibility of assessing longitudinal strain of the human carotid artery using ultrasound speckle tracking needs to be further investigated along with the potential of the method to estimate arterial stiffness and characterize atherosclerotic plaques.

\section{REFERENCES}

[1] M. Catalano, A. Lamberti-Castronuovo, A. Catalano, D. Filocamo, and C. Zimbalatti, "Two-dimensional speckletracking strain imaging in the assessment of mechanical properties of carotid arteries: feasibility and comparison with conventional markers of subclinical atherosclerosis," Eur $J$ Echocardiogr, vol. 12, pp. 528-35, Jul 2011.

[2] H. Hansen, R. Lopata, and C. de Korte, "Noninvasive carotid strain imaging using angular compounding at large beam steered angles: validation in vessel phantoms," IEEE Transactions on Medical Imaging vol. 28, pp. 872-80, 2009.
[3] A. Hoeks, P. Brands, F. Smeets, and R. Reneman, "Assessment of the distensibility of superficial arteries," Ultrasound Med Biol, vol. 16, pp. 121-8, 1990.

[4] C. L. de Korte, M. J. Sierevogel, F. Mastik, C. Strijder, J. A Schaar, E. Velema, G. Pasterkamp, P. W. Serruys, and A. F. van der Steen, "Identification of atherosclerotic plaque components with intravascular ultrasound elastography in vivo: a Yucatan pig study," Circulation, vol. 105, pp. 162730, Apr 92002.

[5] S. Golemati, A. Sassano, M. J. Lever, A. A. Bharath, S. Dhanjil, and A. N. Nicolaides, "Carotid artery wall motion estimated from B-mode ultrasound using region tracking and block matching," Ultrasound Med Biol, vol. 29, pp. 387-99, Mar 2003.

[6] M. Persson, A. R. Ahlgren, T. Jansson, A. Eriksson, H. W. Persson, and K. Lindstrom, "A new non-invasive ultrasonic method for simultaneous measurements of longitudinal and radial arterial wall movements: first in vivo trial," Clin Physiol Funct Imaging, vol. 23, pp. 247-51, Sep 2003.

[7] M. Cinthio, A. R. Ahlgren, J. Bergkvist, T. Jansson, H. W. Persson, and K. Lindstrom, "Longitudinal movements and resulting shear strain of the arterial wall," Am J Physiol Heart Circ Physiol, vol. 291, pp. H394-402, Jul 2006.

[8] G. Zahnd, L. Boussel, A. Marion, M. Durand, P. Moulin, A Serusclat, and D. Vray, "Measurement of two-dimensional movement parameters of the carotid artery wall for early detection of arteriosclerosis: a preliminary clinical study," Ultrasound Med Biol, vol. 37, pp. 1421-9, Sep 2011.

[9] S. Svedlund and L. M. Gan, "Longitudinal common carotid artery wall motion is associated with plaque burden in man and mouse," Atherosclerosis, vol. 217, pp. 120-4, Jul 2011.

[10] S. Svedlund, C. Eklund, P. Robertsson, M. Lomsky, and L. M. Gan, "Carotid artery longitudinal displacement predicts 1-year cardiovascular outcome in patients with suspected coronary artery disease," Arterioscler Thromb Vasc Biol, vol. 31, pp. 1668-74, Jul 2011.

[11] M. Larsson, F. Kremer, P. Claus, T. Kuznetsova, L. A. Brodin, and J. D'Hooge, "Ultrasound-based radial and longitudinal strain estimation of the carotid artery: a feasibility study," IEEE Trans Ultrason Ferroelectr Freq Control, vol. 58, pp. 2244-51, Oct 2011

[12] M. Larsson, F. Kremer, B. Heyde, L.-Å. Brodin, and J. D'hooge, "Ultrasound-based Speckle Tracking for 3D Strain estimation of the Arterial wall - An experimental validation study in a tissue mimicking phantom," in Conference proceedings at IEEE Ultrasonics Symposium, Orlando, USA, 2011.

[13] M. Larsson, F. Kremer, P. Claus, T. Kuznetsova, L.-Å. Brodin, and J. D'hooge, "Ultrasound-based Radial and Longitudinal Strain Estimation of the Carotid Artery: a feasibility study," IEEE Transactions on Ultrasonics, Ferroelectrics, and Frequency Control, vol. 10, pp. 224451., 2011.

[14] O. Lichtenstein, M. E. Safar, P. Poitevin, and B. I. Levy, "Biaxial mechanical properties of carotid arteries from normotensive and hypertensive rats," Hypertension, vol. 26, pp. 15-9, Jul 1995.

[15] P. Tozzi, D. Hayoz, C. Oedman, I. Mallabiabarrena, and L. K. Von Segesser, "Systolic axial artery length reduction: an overlooked phenomenon in vivo," Am J Physiol Heart Circ Physiol, vol. 280, pp. H2300-5, May 2001.

[16] A. R. Ahlgren, M. Cinthio, S. Steen, T. Nilsson, T. Sjoberg, H. W. Persson, and K. Lindstrom, "Longitudinal displacement and intramural shear strain of the porcine carotid artery undergo profound changes in response to catecholamines," Am J Physiol Heart Circ Physiol, vol. 302, pp. H1102-15, Mar 12012. 